\title{
Developing medical ultrasound imaging application across GPU, FPGA, and CPU using oneAPI
}

\author{
Yong, Wang \\ Flex Solutions, Intel Corporation, \\ Beijing, China \\ yong4.wang@intel.com \\ Yang, Wang \\ IAGS, Intel Corporation, Shanghai, \\ China \\ yang.y.wang@intel.com
}

\author{
Yongfa, Zhou \\ Flex Solutions, Intel Corporation, \\ Beijing, China \\ yongfa.zhou@intel.com \\ Qing, Xu \\ IOTG, Intel Corporation, Shanghai, \\ China \\ qing.xu@intel.com
}

\author{
Qi (Scott), Wang \\ IOTG, Intel Corporation, Shanghai, \\ China \\ scott.wang@intel.com \\ Chen, Wang \\ IAGS, Intel Corporation, Shanghai, \\ China \\ chen.wang4@intel.com
}

\begin{abstract}
The Diagnostic ultrasound is a rapidly developing imaging technology that is widely used in the clinic. A typical ultrasound imaging pipeline including the following algorithms: beamforming, Envelope detection, log-compression, and scan-conversion [1]. In tradition, ultrasound imaging is implemented using Application-specific integrated circuits (ASICs) and FPGAs due to its high throughput and massive data processing requirements. With the development of the GPGPU and its programming environments (e.g. CUDA), researchers use software to implement ultrasound imaging algorithms [2], [3].

For now, the two limiting factors of developing ultrasound imaging are: First, using a hardware development approach to implement ultrasound imaging algorithms is complex, time-consuming and lacks flexibility. Second, the existing CUDA-based ultrasound imaging implementations are limited to Nvidia hardware, which is also a restriction applying more architectures.

oneAPI is a cross-platform and unified programming environment developed by intel. It enables heterogeneous computing across multiple hardware architectures using Data Parallel C++ (DPC ++ ). This new programming suite can be used to address the problems mentioned above. To be clear, using a high-level language like $\mathrm{DPC}++$ to program FPGA can accelerate ultrasound imaging application development. SYCL-based ultrasound imaging applications can be easily migrated to other vendor's hardware.

To implement an ultrasound imaging application across multiple architectures (e.g., GPU, FPGA, and CPU) in a unified programming environment. We migrated a CUDA-based open-source ultrasound imaging project SUPRA [4]. The migration process was performed using oneAPI compatibility tool (e.g. dpct). After migration, the code was tuned to run on GPU, FPGA, and CPU.

In this talk, we will discuss our experiences with the complete process of migrating a CUDA code to oneAPI code. First, the whole process of migrating CUDA code base using the $d p c t$ will

Permission to make digital or hard copies of part or all of this work for personal or classroom use is granted without fee provided that copies are not made or distributed for profit or commercial advantage and that copies bear this notice and the full citation on the first page. Copyrights for third-party components of this work must be honored.

For all other uses, contact the owner/author(s).

IWOCL'21, April 27-29, 2021, Munich, Germany

(c) 2021 Copyright held by the owner/author(s).

ACM ISBN 978-1-4503-9033-0/21/04.

https://doi.org/10.1145/3456669.3456680
\end{abstract}

be presented, including usage, code modification, API comparison and build instruction. Second, the ultrasound imaging algorithms' computation characteristics will be analyzed, and we will show how to optimize the application on Intel GPUs, Including ESIDM usage. Third, the early experiences of tuning the migrated code to target FPGA will be highlighted, this will include device code rewrite for FPGA and programming skills to improve performance on FPGA. The device code comparison of GPU and FPGA will also be discussed. Last, we will compare ultrasound imaging algorithms performance and computation results on different hardware, including Intel GPU (integrated GPU and discrete GPU), Intel Arria 10 FPGA, Intel CPU, Nvidia GTX 1080 GPU, and GTX 960M GPU.

\section{CCS CONCEPTS}

- Computing methodologies; • Parallel computing methodologies; • Parallel programming languages;

\section{KEYWORDS}

SYCL, oneAPI, ultrasound imaging, beamforming, migration

\section{ACM Reference Format:}

Yong, Wang, Yongfa, Zhou, Qi (Scott), Wang, Yang, Wang, Qing, Xu, and Chen, Wang. 2021. Developing medical ultrasound imaging application across GPU, FPGA, and CPU using oneAPI. In International Workshop on OpenCL (IWOCL'21), April 27-29, 2021, Munich, Germany. ACM, New York, NY, USA, 1 page. https://doi.org/10.1145/3456669.3456680

\section{ACKNOWLEDGMENTS}

We want to thank the previous work and help from Intel colleagues, Katayama, Takuya; Nakasaka, Masato; Peng, Bo; Wang, Xiaoyun; Wang, Bin; and Zhu, Shaoqing. We also thank the Intel ZiZhu Innovation Funding.

\section{REFERENCES}

[1] T. Y. Phuong and J. G. Lee, "Design space exploration of SW beamformer on GPU," Concurr. Comput. , 2015, doi: 10.1002/cpe.3326.

[2] J. Kang et al., "A System-on-Chip Solution for Point-of-Care Ultrasound Imaging Systems: Architecture and ASIC Implementation," IEEE Trans. Biomed. Circuits Syst., 2016, doi: 10.1109/TBCAS.2015.2431272.

[3] J. W. Choe, A. Nikoozadeh, O. Oralkan, and B. T. Khuri-Yakub, "GPU-based realtime imaging software suite for medical ultrasound," 2013, doi: 10.1109/ULTSYM.2013.0525.

[4] R. Göbl, N. Navab, and C. Hennersperger, "SUPRA: open-source software-defined ultrasound processing for real-time applications: A 2D and 3D pipeline from beamforming to B-mode," Int. F. Comput. Assist. Radiol. Surg., vol. 13, no. 6, pp. 759-767, 2018, doi: 10.1007/s11548-018-1750-6. 